\title{
A METHOD FOR OBTAINING HUMAN SKIN COLOR PIXELS BASED ON SURROUNDING FACTORS
}

\author{
Seok-Woo Jang ${ }^{1}$ and Myoung-Kwan $\mathrm{Oh}^{2 *}$ \\ ${ }^{1}$ Department of Software, Anyang University, Republic of Korea \\ ${ }^{2 *}$ Department of Electrical and Electronic Services, Hyejeon University, \\ Republic of Korea \\ ${ }^{1}$ swjang7285@gmail.com, ${ }^{2 *}$ mkoh@hj.ac.kr
}

\begin{abstract}
Accurate skin color detection methods are very valuably used in diverse fields such as face recognition and tracking, facial expression recognition, adult image detection and healthcare. In this paper, the performances of diverse skin color detection algorithms were comparatively evaluated as the distance to the subject was changed and the color of the subject background was changed in the environment where normal light and indoor lighting were added. The test was conducted by selecting 2 males and 1 female having different skin tones as the subjects and dividing the background colors into white, black, orange, pink and yellow. The skin color extraction algorithms used for performance evaluation were Peer algorithm, NNYUV algorithm, NNHSV algorithm, LutYUV algorithm, and Kismet algorithm. The test was conducted by limiting the distance between camera and subject to $60 \mathrm{~cm}$ to $120 \mathrm{~cm}$. As a result of conducting the performance measurement test, the performances of the algorithms showed differences according to the changes made to the subject background. In general, NNHSV and NNYUV algorithms using neural network and LutYUY algorithm showed stable results. As far as the remaining algorithms were concerned, their skin color detection rates were significantly influenced by the changes made to the background. This paper is expected to be effectively utilized in developing an adaptive and highly accurate environment-based skin color extraction algorithm suitable for actual environments where dynamic changes are made to the subject's surrounding environments.
\end{abstract}

Keywords - Color modeling, Accuracy, Labeling, Dynamic change, Camera

\section{INTRODUCTION}

Recently, a technique accurately detecting human skin is very valuably used in diverse ranges of computer vision-related application fields such as face recognition and tacking, facial expression recognition, gesture analysis, adult image detection, healthcare, medical image analysis and content-based image retrieval [1-4]. The reason for this is because, in such application fields, a search space for detecting a target of interest such as face, hand and particular body part can be considerably reduced through skin region detection. In general, the skin detection process is known to be very effective since it has a small calculation complexity and can be executed regardless of the pose of the subject [5-7].

Most of the pre-existing skin region detection methods used colors to classify pixels of an image into skin color and non-skin colors [8]. This is based on the premise that human skin has a set range of consistent colors differentiated from other various objects.

Received: April 23, 2019

Reviewed: June 27, 2019

Accepted: July 5, 2019

${ }^{*}$ Corresponding Author

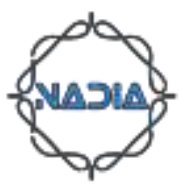


Although this method capable of detecting generally known human skin colors from the entire image is advantageous in that its process is simple, it involves a number of difficulties. Initially, as far as human skin color is concerned, individuals have their own unique skin color, and such skin color is not the same even among each race. Since face, a region utilized the most among all skin regions, is a region exposed to the outside, its skin color may vary depending on special-effect makeup or color makeup. In addition, images of an object can have mutually different colors depending on the characteristics of the optical instruments used for shooting that object or depending on the involved lighting environment. To overcome the described color value differences caused by artificial or natural environmental changes, new methods are being sought diversely.

Skin color detection algorithms must operate with robustness despite the changes made to not only the static environment such as one described above, but also the dynamic environment. For instance, in the case where a camera takes consecutive shots as it moves towards or away from an object or in the case where an object moves in random directions, the color of the moving object will be consistently maintained to a certain extent, but its background will be changed continuously. Therefore, this may have a negative influence on the performance of skin color detection. Namely, skin color detection methods must achieve a good performance even in an actual environment where the distance between camera and subject is not fixed and the background color changes dynamically. However, pre-existing skin color detection methods are mainly focused on static environmental changes, and there are only a limited number of studies focusing on such dynamic environmental changes.

Accordingly, in this paper, the performances of diverse skin color detection algorithms were comparatively analyzed through examining how the subject skin detection rate changed as the distance between camera and subject changed and the color of the subject background changed in the environment where normal light and indoor lighting were added. In this paper, the test was conducted by selecting 2 males and 1 female having different skin tones as the subjects and dividing the background colors into white, black, orange, pink and yellow. The pre-existing skin color detection algorithms used for performance evaluation were Peer algorithm [9], NNYUV, NNHSV, LutYUV, and Kismet algorithm [10]. The test was conducted by limiting the distance between camera and subject to $60 \mathrm{~cm}$ to $120 \mathrm{~cm}$. The purpose of this was to diversely analyze the algorithms that showed a good performance despite the changes made to the dynamic environment, and, thereby, develop a skin color detection system maintaining its robustness despite the changes made to not only the static environment, but also the dynamic environment, in the future.

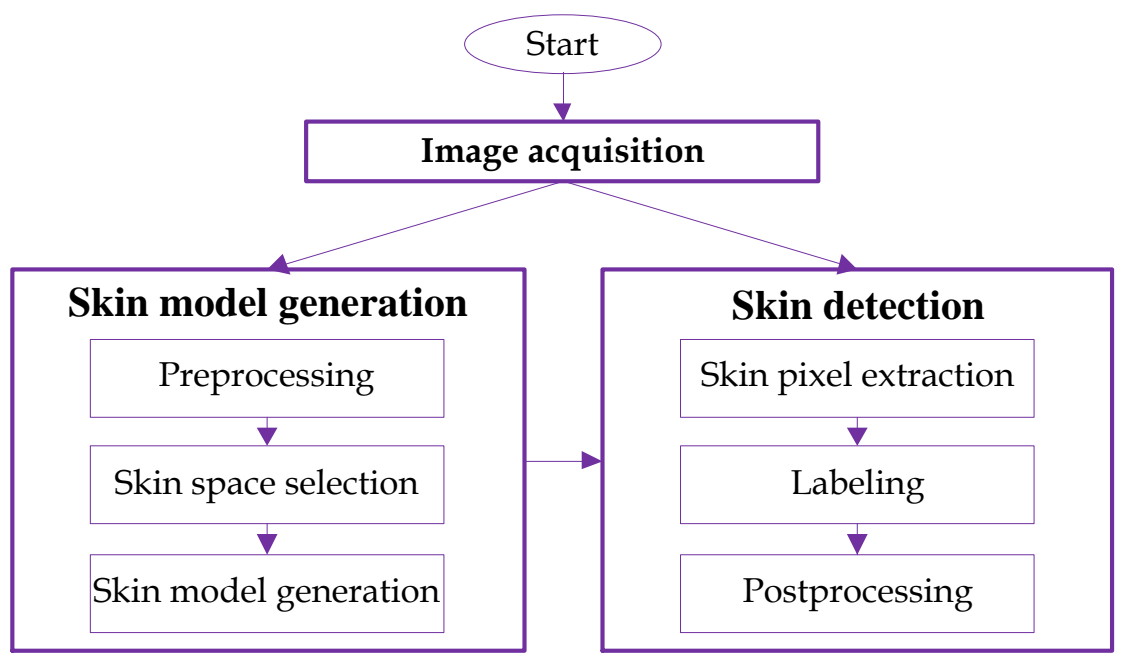

Fig. 1 Overall flow of the system 
In general, the overview diagram of the skin color detection algorithms is as shown in Figure 1. Initially, an input image is received, and the quality of the input image is enhanced through pre-processing stages such as smoothing. Then, a color space to be used by the algorithm is selected, and either a skin color model is manufactured or a preexisting model is selected to be used for skin color detection. Once the acquired skin model is used to extract the pixels determined to be skin color from the image, the individual skin pixels are connected through labeling and grouped into regional units. Once post-processing stages are executed to remove noises or regions very small in size, the finally extracted skin color regions are detected.

Section 1 described the motivation, background, and overall outline of the study. In Section 2, we introduce the existing skin color detection algorithms. In Section 3, we describe the main components of the skin color extraction method. Section 4 presents various experimental results. Finally, Section 5 presents conclusions and future research directions.

\section{RELATED WORK}

Since HCI (Human Computer Interaction) is being magnified as an important issue and a technique robustly detecting human skin color is being used as core technology in a number of related application fields, a number of related studies are being conducted recently.

Peer detected facial regions from an image using, in 2D and 3D spaces, a skin color clustering method non-sensitive to the lighting conditions and noises involved in the input image shooting [9]. As far as this method was concerned, detecting facial regions just based on color information was more effective when clustering was used in a 3D color space, and detecting facial regions based on other characteristics-based methods along with color information was more effective when clustering was used in a $2 \mathrm{D}$ color space. In the perspective of lighting, the method used in a $2 \mathrm{D}$ color space reduced the influence of noises more effectively on a dark image. However, its performance was lowered as it detected too many pixels as skin color on an image shot in a normal daytime lighting environment. In addition, in order to use this method to robustly extract the skin color regions in a non-standard lighting environment, independent algorithms were added to such lighting.

An artificial intelligence research laboratory located within Massachusetts Institute of Technology, used a robot capable of expressing emotions to mimic human's facial expressions to naturally interact with humans, and eventually developed Kismet [10]. Kismet is equipped with a system capable of not only naturally recognizing signals through visual and voice information such as facial expression, line of vision, hand gesture and color brightness, but also using a method identical to that of humans to deliver signals. Kismet extracts skin color from an input image through suggesting a skin tone feature map from diverse lighting conditions and skin color pixels. As a result of using this method, the skin tone filter responded that approximately $4.7 \%$ of the usable R, $\mathrm{G}, \mathrm{B}$ values were skin color.

Lee proposed a new skin region extraction method for adult image detection [11]. Initially, an image of the RGB color model was converted into the $\mathrm{YCbCr}$ color model, and a histogram displaying the distribution of $\mathrm{Cb}$ and $\mathrm{Cr}$ was prepared and combined with the converted model to manufacture a skin color model for skin color detection. As far as this method was concerned, to resolve the problem of different skin regions being detected according to the overall image brightness and color, skin colors being distributed differently according to the image brightness were classified, and a number of skin color models were created. Using such created skin color models, the overall image was divided into object regions and background regions. This method was disadvantageous in that the background regions sharing a color similar to skin color were miscalculated as a 
skin region. To improve such problem, the overall image was divided into sub-regions for energy detection, and any sub-region having an energy level above a certain threshold value was determined to be non-skin. This method was mainly used for images shot under special lighting.

Cho proposed a method capable of adaptively adjusting threshold values instead of using a fixed threshold value to distinguish between skin color and non-skin color [8]. This method largely consisted of 2 main phases. In the initial phase, the RGB color space was converted into the HSV color space, and 2 threshold values were set for each color factor to configure a 3D threshold value box. Then, while the $\mathrm{H}$ value was fixed, a color histogram was used to adaptively adjust the 3D threshold value box. This process was executed under the assumption that, on an image, the region taken up by skin color will be greater than the region taken up by similar color on an image. In the second phase, by dividing the color vectors within the 3D threshold value box into skin color vectors and non-skin color vectors, it was possible to extract from an image the pixels determined to be skin color. This method was capable of effectively detecting the skin color region through adaptively adjusting threshold values. However, this method was limited in that the regions determined to be skin color had to be above a certain size on the overall image, and was disadvantageous in that it does not operate well when lighting is strong.

In addition, more methods can be found [12-14]. Fang proposed a method capable of using a new color histogram for skin color detection. This method effectively detected facial regions through preparing a color histogram within regions determined to be a facial region and creating a vector reflecting the spatial relationship with facial regions [12]. Kawaguchi proposed an algorithm capable of detecting the iris of two eyes from a facial image [13]. They roughly extracted facial regions using edges, geometrical characteristics and skin color models, and then detected the iris from the extracted facial regions. However, this method was disadvantageous in that it was unable to precisely detect the boundary of facial regions due to use of a non-exquisite geometrical measuring scale. Sigal proposed a method capable of dividing skin regions from video data on a real-time basis [14]. To predict how the skin color histogram progresses as time progresses, the secondary Markov model was selected. This method was particular in that the two models share a temporal correlation since the current skin color distribution updates the past model.

\section{COLOR SPACE-BASED SKIN MODELING}

Skin color detection algorithms consist of a number of component factors. Among these component factors, factors used for determining color space and modeling skin color are the most important factors [15]. In other words, the overall skin color extraction performance is greatly influenced by the determined color space and modeled skin color.

RGB color space is the most well-known model, and it combines the three color components of R, G and B to display colors conveniently. However, since this model includes channels that share a high correlation and its color and brightness values are rather combined than separated, it is not frequently applied to algorithms used for color analysis and color-based recognition.

Normalized RGB color space can be easily expressed, as shown in Equation (1), from the RGB space through a simple normalization process. In this color space, since the sum of the three normalized color factors is known $(\mathrm{r}+\mathrm{g}+\mathrm{b}=1)$, the third factor has no important significance. Accordingly, it is possible to omit the third color factor, and as a result, the color dimension is reduced from calculating $3 \mathrm{D}$ to calculating $2 \mathrm{D}$.

$$
r=\frac{R}{R+G+B}, \quad g=\frac{G}{R+G+B}, \quad b=\frac{B}{R+G+B}
$$


HSV color space represents hue, saturation and valve. Since this color space is most similar to how humans sense colors, and has separated hue, saturation and value, it is frequently applied to various color processing operations. This color space cannot be directly expressed from R, G, and B, and is expressed as shown in Equation (2).

$$
\begin{aligned}
& H=\cos ^{-1}\left(\frac{[(R-G)+(R-B)]}{2 \sqrt{(R-G)^{2}+(R-B)(G-B)}}\right) \\
& S=1-\frac{3}{(R+G+B)} \times[\min (R, G, B)] \\
& V=\frac{1}{3} \times(R+G+B)
\end{aligned}
$$

$\mathrm{YC}_{\mathrm{b}} \mathrm{C}_{\mathrm{r}}$ color space is a space frequently used in the European television studios as well as in the field of image compression, and is an encoded, non-linear RGB signal. Since this space has separated color and brightness values, it is frequently used in the fields of color division and skin color detection. $\mathrm{YC}_{\mathrm{b}} \mathrm{C}_{\mathrm{r}}$ color space, as shown in Equation (3), is expressed with color brightness value and hue. $\mathrm{Y}$ representing brightness value is calculated through calculating the weighted sum of $R, G$ and $B$ values, and $C_{b}$ and $C_{r}$ representing hue are calculated through subtracting $\mathrm{Y}$, the brightness value factor, from the factors of $\mathrm{B}$ and $\mathrm{R}$.

$$
\begin{aligned}
& Y=0.299 \times R+0.587 \times B+0.114 \times B \\
& C_{r}=R-Y \\
& C_{b}=B-Y
\end{aligned}
$$

In addition to the color spaces described above, there are many space models such as YIQ, TSL, CIE, YES, CMY, and CIELab color model.

To effectively detect skin color in a given color space is the process of determining whether given pixels are skin pixels or non-skin pixels. Namely, the ultimate purpose of a skin color extraction method is to model a measure that can be used to accurately distinguish between skin pixels and non-skin pixels. Methods using a 2D or 3D histogram express the distribution of skin color in a given space through histogram. In other words, a color space is quantized into a number of histogram bins, and each histogram bin (can be defined as a look-up table cell) saves the frequency of related colors. The frequency considered a histogram bin is converted based on the following probability distribution. In Equation (4), count(c) represents the number of histogram bins considered color c, and $\mathrm{T}$ is the sum of all histogram bins. Since $\mathrm{P}(\mathrm{c})$ is the possibility of a given color being considered skin color, the threshold value can be used to distinguish between skin color and non-skin color.

$$
P(c)=\frac{\operatorname{count}(c)}{T}
$$

Methods using a neural network to robustly model skin color, in general, use multi-layer perceptron to learn skin colors and non-skin colors from learning data. In these methods, the number of middle layers, the number of middle nodes, and the learning rate of the neural network must be determined to effectively learn skin 
colors. The weights in the network are iteratively updated through a gradient descent technique known as back-propagation algorithm.

Methods using an elliptic boundary model are methods using the nature of skin colors being distributed within an ellipse-shaped boundary in a given color space. In these methods, an ellipse is positioned at the spot where skin colors are expected to be distributed in a color space to model skin colors in an ellipse-shape, and parameters such as central location of ellipse and length/angle of minor/major axes are extracted to allow an ellipse to fit into the skin color boundary with the closest approach. Once the above procedures are complete and a skin color model is created, a test image is input and those parameters are used to detect skin colors.

In addition to the skin color model algorithms described above, there are methods using Gaussian Classifier, SOM (Self Organizing Map) Classifier, Bayesian Network, and HMM [16-19].

\section{EXPERIMENTAL RESULTS}

In this paper, the computer used for the test was Intel Pentium Core 2 Duo CPU Processor (4GB of Memory), and the operating system used was Microsoft's Windows 7 Professional. The program used for manufacturing the program was Visual $\mathrm{C}++$, and the camera used for the test was Lebeca Web Camera manufactured by PANWEST.

In this paper, the algorithms used for comparatively evaluating the performances of skin color detection methods were Peer algorithm [9], Kimset algorithm [10], NNYUV algorithm, NNHSV algorithm, and LutYUV algorithm. Peer algorithm and Kismet algorithm, as mentioned in Section 2, were manufactured into applications and were skin color detection algorithms selected to be used on an actual system currently in use. NNYUV and NNHSV algorithms were developed in this paper to distinguish between skin colors and non-skin colors through using multi-layer neural network that provides high accuracy in the YUV and HSV color spaces serving as the two most frequently used color spaces among all color spaces. In addition, LutYUV algorithm was manufactured to use a look-up table in the YUV color space for efficient color clustering.

In this paper, the test was conducted by limiting the distance between camera and subject to $60 \mathrm{~cm}$ to $120 \mathrm{~cm}$. In addition, 2 males and 1 female having different color tones were selected as the subjects, and the background colors were divided into white, black, orange, pink and yellow. The purpose of dividing the background color into 5 different colors was to find out what influence background colors have on skin color detection.

Figure 2 shows the structure of the main program developed to test the performance of the skin color detection algorithms described above, and it was programmed in a way that the skin color extraction results of an input image achieved from various algorithms can be confirmed simultaneously. In Figure 2, the upper left window shows an instantaneously captured still image of the image input from the camera, and the lower left window consecutively shows the real-time images input from the camera. The right window simultaneously shows the test result images achieved from the skin color detection algorithms. 


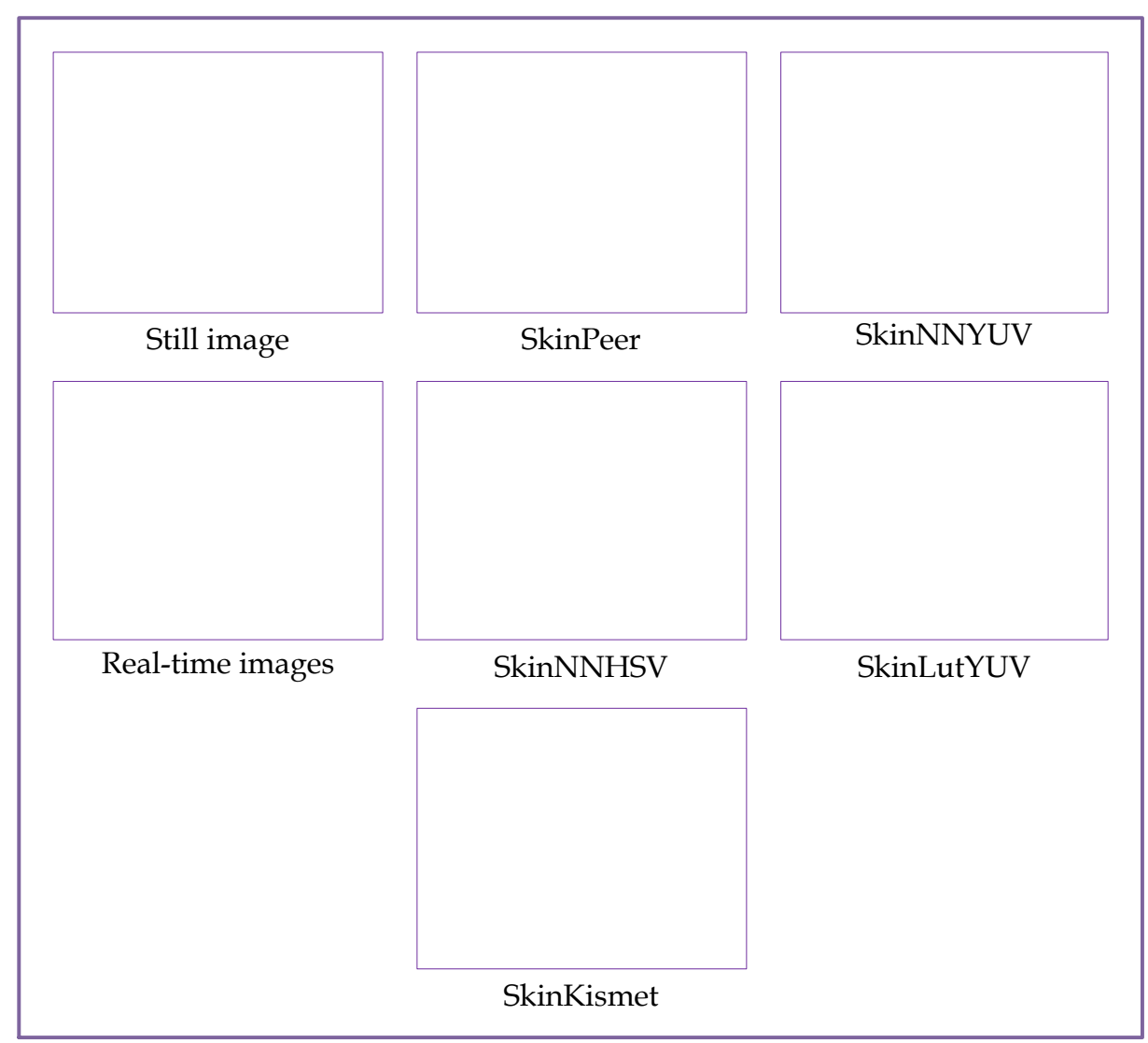

Fig. 2 Structure of main program

In this paper, in order to analyze the performances of the skin color detection algorithms, how well each algorithm detects the skin colors displayed on the original image was examined. As far as the skin color detection rate was concerned, the ground truth of skin pixels of the original image was extracted, and the number of skin color pixels detected by each algorithm was calculated. In this paper, ground truth refers to the scope of skin color of the original image, and the data was manually acquired through Photoshop. Equation (5) was used to calculate each algorithm's skin color detection rate $D_{\text {rate. }}$ In Equation (5), $\mathrm{N}_{\text {skin }}(\mathrm{TI})$ represents the number of pixels extracted as skin color after each algorithm was applied, and $\mathrm{N}_{\mathrm{GT}}(\mathrm{OI})$ represents the number of ground truth pixels of the skin colors displayed on the original image.

$$
D_{\text {rate }}=\frac{N_{\text {skin }}(T I)}{T_{G T}(O I)} \times 100(\%)
$$

Table 1 shows one part of the skin color detection results achieved in the environment where the subject's background was set to black and the normal light and indoor lighting had an illumination intensity of 350lux. In Table $1, \mathrm{BG}_{\text {rate }}$ represents the incorrect recognition rate of the regions excluding the ground truth of the original image, $\mathrm{FG}_{\text {rate }}$ represents the rate taken up by the ground truth on the original image, and $\mathrm{O}_{\text {pixel }}$ represents the number of pixels considered the ground truth of the original image.

As Table 1 shows, in the case where the subject's background was set to black, outstanding results were achieved from Peer algorithm and Kismet algorithm. In addition to such results shown in Table 1 , in the case where the distance between camera and subject was set to a value not exceeding $320 \mathrm{~cm}$, the numbers confirmed that these two algorithms provided better results than the other three algorithms. As shown in Table I, 
the reason that Kismet algorithm's $\mathrm{D}_{\text {rate }}$ was above 100 is because, the clothes and background, excluding the ground truth of skin pixels of the original image, were incorrectly recognized. In particular, as the distance to the subject got closer to $320 \mathrm{~cm}$, the incorrect recognition rate increased rapidly due to the background.

Table I. Skin Color Extraction (Background: Black)

\begin{tabular}{|c|c|c|c|c|c|}
\hline Distance & Algorithm & $\mathrm{D}_{\text {rate }}$ & $\overline{\mathrm{BG}_{\text {rate }}}$ & $\mathrm{FG}_{\text {rate }}$ & $\mathrm{O}_{\text {pixel }}$ \\
\hline \multirow{5}{*}{60} & SkinPeer & 75.65 & \multirow{5}{*}{3.42} & \multirow{5}{*}{22.22} & \multirow{5}{*}{17066} \\
\hline & SkinNNYUV & 9.32 & & & \\
\hline & SkinNNHSV & 46.62 & & & \\
\hline & SkinLutYUV & 43.72 & & & \\
\hline & SkinKismet & 111.98 & & & \\
\hline \multirow{5}{*}{70} & SkinPeer & 71.84 & \multirow{5}{*}{1.46} & \multirow{5}{*}{18.21} & \multirow{5}{*}{13983} \\
\hline & SkinNNYUV & 8.65 & & & \\
\hline & SkinNNHSV & 51.91 & & & \\
\hline & SkinLutYUV & 40.23 & & & \\
\hline & SkinKismet & 106.58 & & & \\
\hline \multirow{5}{*}{80} & SkinPeer & 78.97 & \multirow{5}{*}{2.65} & \multirow{5}{*}{14.17} & \multirow{5}{*}{10882} \\
\hline & SkinNNYUV & 10.91 & & & \\
\hline & SkinNNHSV & 54.38 & & & \\
\hline & SkinLutYUV & 41.22 & & & \\
\hline & SkinKismet & 116.07 & & & \\
\hline
\end{tabular}

Table II separately shows the mean value of $\mathrm{D}_{\text {rate }}$ achieved by each recognition algorithm when the subject's background was black. In Table 2, mean value a is the mean value of $D_{\text {rate }}$ for all tested distances, and mean value $b$ is the mean value of $D_{\text {rate }}$ when the distance limitation was set to $120 \mathrm{~cm}$ to decrease the incorrect recognition rate influenced by the background. In this paper, to distinguish between subject $A$ and subject $B$, it is clarified that subject $\mathrm{A}$ is a male with relatively bright skin, subject $\mathrm{B}$ is a male with dark skin, and subject $\mathrm{C}$ is a female wearing makeup. As shown in Table II, Peer algorithm showed the most ideal results.

Table II. Skin Color Extraction (Background: Black2)

\begin{tabular}{|c|c|c|c|c|c|c|}
\hline & \multicolumn{2}{|c|}{ Target A } & \multicolumn{2}{c|}{ Target B } & \multicolumn{2}{c|}{ Target C } \\
\cline { 2 - 7 } & average a & average b & average a & average b & average a & average b \\
\hline SkinPeer & 101.27 & 76.81 & 131.20 & 71.31 & 126.36 & 86.47 \\
\hline SkinNNYUV & 20.12 & 10.43 & 44.02 & 22.28 & 39.45 & 27.40 \\
\hline SkinNNHSV & 73.38 & 55.02 & 123.02 & 77.80 & 110.12 & 85.19 \\
\hline SkinLutYUV & 42.42 & 41.70 & 68.24 & 58.64 & 58.65 & 53.71 \\
\hline SkinKismet & 172.68 & 118.86 & 288.86 & 139.05 & 280.78 & 165.86 \\
\hline
\end{tabular}

In the case where the background was set to orange, Kismet algorithm achieved satisfactory results through detecting almost all skin colors included in the ground truth of the original image, but a $D_{\text {rate }}$ value higher than necessary was achieved because the algorithm detected regions other than skin as well. In particular, Peer algorithm managed to detect skin regions, but also detected the background itself as a skin region. As far as LutYUV, NNHSV and NNYUV algorithms were concerned, $D_{\text {rate }}$ for the ground truth was low in comparison to Kismet algorithm, but the detection rate for the clothes and background, excluding skin colors, was much lower in comparison to Kismet algorithm, 
meaning that these algorithms among the five algorithms showed the most outstanding effects in the case where orange, a color closest to skin color, was used as the background. Table 3 shows the test results achieved in the case where the background was set to orange. As the distance between subject and camera increases, more background regions become detected and this increases the mean value of $\mathrm{D}_{\text {rate }}$. Peer algorithm is a good example of this. As the distance between subject and camera exceeded $140 \mathrm{~cm}$, Peer algorithm detected the background itself as skin. In the case where the background was set to orange, the algorithm's detection rate for the clothes increased.

Table III. Skin Color Extraction (Background: Orange)

\begin{tabular}{|c|c|c|c|c|c|c|}
\hline & \begin{tabular}{c}
$\mathbf{D}_{\text {rate }}$ \\
\cline { 2 - 7 }
\end{tabular} & $\begin{array}{c}\mathbf{B G}_{\text {rate }} \\
\text { average a }\end{array}$ & $\begin{array}{c}\mathbf{F G}_{\text {rate }} \\
\text { average b }\end{array}$ & $\begin{array}{c}\mathbf{F G}_{\text {rate }} \\
\text { average a }\end{array}$ & $\begin{array}{c}\text { Opixel } \\
\text { average b }\end{array}$ & $\begin{array}{c}\text { Opixel }^{\text {average a }} \\
\text { average b }\end{array}$ \\
\hline SkinPeer & 1029.77 & 699.20 & 1495.45 & 802.97 & 1121.52 & 635.77 \\
\hline SkinNNYUV & 39.44 & 15.88 & 75.26 & 51.85 & 60.07 & 38.95 \\
\hline SkinNNHSV & 94.34 & 61.71 & 157.29 & 113.60 & 156.37 & 156.85 \\
\hline SkinLutYUV & 55.09 & 39.96 & 96.64 & 82.35 & 76.29 & 69.05 \\
\hline SkinKismet & 309.74 & 168.34 & 396.44 & 169.25 & 372.27 & 230.95 \\
\hline
\end{tabular}

In the case where the subject's background was set to pink, the results were significantly different among LutYUV algorithm, NNHSV and NNYUV algorithms using neural network, and the remaining algorithms. The algorithm described above detected skin colors only from the ground truth of the original image, whereas Peer algorithm and Kismet algorithm detected not only the ground truth but also pink, the background color, as skin colors. Due to this, the mean value of $\mathrm{D}_{\text {rate }}$ increased exponentially. The test results achieved while the background was set to pink were as shown in Table IV. In this paper, mean value a is the mean value of $D_{\text {rate }}$ for all images having a test distance ranging from $60 \mathrm{~cm}$ to $320 \mathrm{~cm}$, and mean value $b$ is the mean value of $D_{\text {rate }}$ for all images having a close distance not exceeding $120 \mathrm{~cm}$. It was found that LutYUV algorithm showed the most outstanding results.

Table IV. Skin Color Extraction (Background: Pink)

\begin{tabular}{|c|c|c|c|c|c|c|}
\hline & $\mathbf{D}_{\text {rate }}$ & $\mathbf{B G}_{\text {rate }}$ & $\mathbf{F G}_{\text {rate }}$ & $\mathbf{F G}_{\text {rate }}$ & $\mathbf{O}_{\text {pixel }}$ & $\mathbf{O}_{\text {pixel }}$ \\
\cline { 2 - 7 } & average a & average b & average a & average b & average a & average b \\
\hline SkinPeer & 1195.05 & 855.21 & 1544.50 & 812.45 & 1111.97 & 703.50 \\
\hline SkinNNYUV & 76.21 & 31.39 & 121.37 & 63.63 & 88.59 & 49.58 \\
\hline SkinNNHSV & 132.82 & 76.27 & 208.82 & 132.19 & 164.52 & 132.50 \\
\hline SkinLutYUV & 89.30 & 61.10 & 118.51 & 85.44 & 95.58 & 71.40 \\
\hline SkinKismet & 1283.43 & 885.69 & 1724.37 & 906.21 & 1228.29 & 795.97 \\
\hline
\end{tabular}

In the case where the subject background was set to yellow, similar to the case where the background was set to pink, the results were significantly different among LutYUV algorithm, NNHSV and NNYUV algorithms using neural network, and the remaining algorithms. LutYUV, NNHSV and NNYUV algorithms mentioned above detected skin colors only from the ground truth of the original image, whereas Peer algorithm and Kismet algorithm, detected not only the ground truth but also pink, the background color, as skin colors, as the distance between subject and camera increased. Due to this, the value of Drate increased as the distance between subject and camera increased. Table V 
shows the skin color detection rate achieved in the case where the subject's background was set to yellow.

Table V. Skin Color Extraction (Background: Yellow)

\begin{tabular}{|c|c|c|c|c|c|c|}
\hline & $\mathbf{D}_{\text {rate }}$ & $\mathbf{B G}_{\text {rate }}$ & $\mathbf{F G}_{\text {rate }}$ & $\mathbf{F G}_{\text {rate }}$ & $\mathbf{O}_{\text {pixel }}$ & $\mathbf{O}_{\text {pixel }}$ \\
\cline { 2 - 7 } & average a & average b & average a & average b & average a & average b \\
\hline SkinPeer & 668.86 & 194.96 & 611.66 & 270.92 & 473.11 & 190.99 \\
\hline SkinNNYUV & 45.76 & 8.87 & 39.85 & 18.90 & 39.26 & 16.60 \\
\hline SkinNNHSV & 117.90 & 53.11 & 123.84 & 73.76 & 100.82 & 66.26 \\
\hline SkinLutYUV & 62.97 & 37.42 & 68.01 & 56.04 & 59.51 & 40.57 \\
\hline SkinKismet & 599.03 & 150.44 & 469.92 & 246.16 & 384.57 & 207.92 \\
\hline
\end{tabular}

In the case where the subject's background was set to yellow, Peer algorithm and Kismet algorithm showed significantly outstanding detection rates for the ground truth of the original image. LutYUV, NNHSV and NNYUV algorithms achieved high skin color detection result values without being particularly influenced by the background.

In the case where the subject's background was set to white, Peer algorithm and Kismet algorithm showed outstanding detection rates for the ground truth of the original image. LutYUV, NNHSV and NNYUV algorithms achieved high skin color detection result values without being particularly influenced by the background. However, Kismet and Peer algorithms showed a phenomenon where they started to recognize the white background as skin as the distance between camera and subject exceeded $290 \mathrm{~cm}$.

As a result, the performances of the algorithms showed differences as changes were made to the subject's background. Overall, NNHSV and NNYUV algorithms using neural network and LutYUV algorithm showed stable results. Kismet algorithm showed the most outstanding recognition rate for the ground truth of the original image, but its skin color detection rate was significantly influenced by pink and yellow sharing a tone similar to skin ton, and this considerably contributed to increasing its incorrect recognition rate. In addition, Peer algorithm was the one most influenced by the background. In particular, similar to Kismet algorithm, it recognized the background as skin in the case where the background was set to pink, yellow and orange, and this significantly contributed to increasing its incorrect recognition rate.

Table VI. Skin Color Extraction (Background: White)

\begin{tabular}{|c|c|c|c|c|c|c|}
\hline & $\mathbf{D}_{\text {rate }}$ & $\mathbf{B G}_{\text {rate }}$ & $\mathbf{F G}_{\text {rate }}$ & $\mathbf{F G}_{\text {rate }}$ & $\mathbf{O}_{\text {pixel }}$ & $\mathbf{O}_{\text {pixel }}$ \\
\cline { 2 - 7 } & average a & average b & average a & average b & average a & average b \\
\hline SkinPeer & 165.09 & 90.90 & 158.18 & 72.98 & 161.39 & 84.30 \\
\hline SkinNNYUV & 50.29 & 19.00 & 75.06 & 42.08 & 48.77 & 28.50 \\
\hline SkinNNHSV & 106.66 & 65.87 & 167.92 & 94.51 & 108.82 & 80.44 \\
\hline SkinLutYUV & 65.15 & 45.27 & 86.19 & 67.12 & 66.05 & 54.12 \\
\hline SkinKismet & 244.96 & 108.42 & 310.48 & 131.06 & 240.35 & 133.52 \\
\hline
\end{tabular}

\section{CONCLUSION}

In this paper, the performances of diverse skin color detection algorithms were comparatively analyzed through examining how the subject skin detection rate changed as the distance between camera and subject changed and the color of the subject background changed in the environment where normal light and indoor lighting were added. The purpose of this was to diversely analyze the skin color extraction algorithms that showed a good performance despite the changes made to the dynamic environment, 
and, thereby, develop a skin color detection system maintaining its robustness despite the changes made to not only the static environment but also the dynamic environment, in the future.

The skin color extraction algorithms used for performance evaluation were Peer algorithm, NNYUV algorithm, NNHSV algorithm, LutYUV algorithm, and Kismet algorithm. The test was conducted by limiting the distance between camera and subject to $60 \mathrm{~cm}$ to $120 \mathrm{~cm}$. The test was conducted by selecting 2 males and 1 female having different skin tones as the subjects and dividing the background colors into white, black, orange, pink and yellow. As a result of conducting diverse tests, it was found that the performances of the algorithms varied depending on the subject's background. Overall, NNHSV and NNYUV algorithms using neural network and LutYUV algorithm showed stable results. Kismet algorithm and Peer algorithm showed outstanding recognition rates for the ground truth of the original image, but the background set to pink and yellow sharing a tone similar to skin tone had a negative influence on their skin color detection rates.

In the future, it is scheduled to develop a system capable of robustly detecting skin colors in general indoor and outdoor environments where the subject's background color changes dynamically. To do so, it is scheduled to develop a mechanism capable of adaptively selecting, case by case, a skin color detection method suitable for such dynamic environments based on the performance evaluation data achieved through this paper, and it is also scheduled to propose a scale for automation of such process. In addition, it is scheduled to conduct a research to propose an objective index that can be used to compare the performances of the algorithms according to the changes made to their surrounding environments.

\section{ACKNOWLEDGMENTS}

This research was supported by Basic Science Research Program through the National Research Foundation of Korea (NRF) funded by the Ministry of Education (2016R1D1A1A09917838).

\section{REFERENCES}

[1] Krishnan, K. S., Saha, A., Ramachandran, S., Kumar, S. "Recognition of Human Arm Gestures Using Myo Armband for the Game of Hand Cricket," IEEE International Symposium on Robotics and Intelligent Sensors, Ottawa, Canada, 5-7, October 2017, pp. 389-394.

[2] Jang, S.-W., Lee, G.-J., Jung, M. "Effective Detection of Target Region Using a Machine Learning Algorithm," Journal of Korea Academia-Industrial Cooperation Society 19.5 (2018): 697-704.

[3] Hwang, D., Lee, K. "Robust Skin Area Detection Method in Color Distorted Images," Journal of Korea Academia-Industrial Cooperation Society 18.7 (2017): 350-356.

[4] Hong, D. "Correlation between Interests of Hand's Health and Hand Skin Care Behaviors by Age Group,” Journal of Korea Academia-Industrial Cooperation Society 18.11 (2017): 158-165.

[5] Phung, S. L., Bouzerdoum, A., Chai, D. "Skin Segmentation Using Color Pixel Classification: Analysis and Comparison," IEEE Transactions on Pattern Analysis and Machine Intelligence 27.1 (2005): 148154.

[6] Zakaria, Z., Suandi, S. A., Mohamad-Saleh, J. "Hierarchical Skin-AdaBoost-Neural Network (HSKANN) for Multi-Face Detection," Applied Soft Computing 68 (2018): 172-190.

[7] Brancati, N., Pietro, G. D., Frucci, M., Gallo, L. "Human Skin Detection through Correlation Rules between the YCb and YCr Subspaces Based on Dynamic Color Clustering," Computer Vision and Image Understanding 155 (2017): 33-42.

[8] Cho, K.-M., Jang, J.-H., Hong, K.-S. “Adaptive Skin-Color Filter,” Pattern Recognition 34.5 (2001): 1067-1073.

[9] Kovac, J., Peer, P., Solina, F. "Human Skin Color Clustering for Face Detection," International Conference on Computer as a Tool 2 (2003): 144-1448.

[10] Breazeal, C., Edsinger, A., Fitzpatrick, P., Scassellati, B. “Active Vision for Sociable Robots," IEEE Transactions on Systems, Man, and Cybernetics-Part A: Systems and Humans 31.5 (2001): 443-453.

[11] Lee, J.-S., Kuo, Y.-M., Chung, P.-C., Chen, E.-L. "Naked Image Detection based on Adaptive and Extensible Skin Color Model," Pattern Recognition 40.8 (2007): 2261-2270. 
[12] Fang J., Qiu, G. “A Color Histogram-based Approach to Human Face Detection," International Conference on Visual Information Engineering, Guildford, UK, 7-9, July 2003, pp. 133-136.

[13] Kawaguchi T., Rizon, M. "Iris Detection Using Intensity and Edge Information," Pattern Recognition 36.2 (2003): 549-562.

[14] Sigal, L., Sclaroff, S., Athitsos, V. "Skin Color-based Video Segmentation under Time-Varying Illumination," IEEE Transactions on Pattern Analysis and Machine Intelligence 26.7 (2004): 863-877.

[15] Cernadas, E., Fernandez-Delgado, M., Gonzalez-Rufino, E., Carrion, P. "Influence of Normalization and Color Space to Color Texture Classification,” Pattern Recognition 61 (2017): 120-138.

[16] Wang, S.-C., Gao, R., Wang, L.-M. "Bayesian Network Classifiers Based on Gaussian Kernel Density," Expert Systems with Applications 51 (2016): 207-217.

[17] Hoz, E. D. L., Hoz, E. D. L., Ortiz, A., Ortega, J., Prieto, B. "PCA Filtering and Probabilistic SOM for Network Intrusion Detection,” Neurocomputing 164 (2015): 71-81.

[18] Sun, Y., Tang, Y., Ding, S., Lv, S., Cui, Y. "Diagnose the Mild Cognitive Impairment by Constructing Bayesian Network with Missing Data,” Expert Systems with Applications 38.1 (2011): 442-449.

[19] Saini, R., Roy, P. P., Dogra, D. P. "A Segmental HMM based Trajectory Classification Using Genetic Algorithm," Expert Systems with Applications 93 (2018): 169-181. 